OPEN ACCESS

Edited by:

Cesare Mancuso,

Catholic University of the Sacred

Heart, Italy

Reviewed by:

Ileana Soto,

Rowan University, United States

Claes Wahlestedt,

University of Miami, United States

*Correspondence:

Silvia Di Angelantonio silvia.diangelantonio@uniroma1.it; silvia.diangelantonio@iit.it

Specialty section: This article was submitted to

Neuropharmacology,

a section of the journal

Frontiers in Neuroscience

Received: 15 May 2019

Accepted: 19 August 2019

Published: 04 September 2019

Citation:

Grimaldi A, Pediconi N, Oieni F, Pizzarelli R, Rosito M, Giubettini M,

Santini T, Limatola C, Ruocco G,

Ragozzino $D$ and Di Angelantonio $S$ (2019) Neuroinflammatory Processes,

A1 Astrocyte Activation and Protein Aggregation in the Retina of Alzheimer's Disease Patients,

Possible Biomarkers for Early

Diagnosis. Front. Neurosci. 13:925. doi: 10.3389/fnins.2019.00925

\section{Neuroinflammatory Processes, A1 Astrocyte Activation and Protein Aggregation in the Retina of Alzheimer's Disease Patients, Possible Biomarkers for Early Diagnosis}

\author{
Alfonso Grimaldi', Natalia Pediconi', Francesca Oieni' ${ }^{2}$, Rocco Pizzarelli', Maria Rosito', \\ Maria Giubettini ${ }^{3}$, Tiziana Santini ${ }^{1}$, Cristina Limatola ${ }^{2,4}$, Giancarlo Ruocco ${ }^{1,5}$, \\ Davide Ragozzino ${ }^{2,4}$ and Silvia Di Angelantonio ${ }^{1,2 *}$

\begin{abstract}
' Center for Life Nanoscience, Istituto Italiano di Tecnologia, Rome, Italy, ${ }^{2}$ Department of Physiology and Pharmacology, Sapienza University, Rome, Italy, ${ }^{3}$ Crestoptics S.p.A., Rome, Italy, ${ }^{4}$ IRCCS Neuromed, Pozzilli, Italy, ${ }^{5}$ Department
\end{abstract} \\ of Physics, Sapienza University, Rome, Italy
}

Alzheimer's disease (AD), a primary cause of dementia in the aging population, is characterized by extracellular amyloid-beta peptides aggregation, intracellular deposits of hyperphosphorylated tau, neurodegeneration and glial activation in the brain. It is commonly thought that the lack of early diagnostic criteria is among the main causes of pharmacological therapy and clinical trials failure; therefore, the actual challenge is to define new biomarkers and non-invasive technologies to measure neuropathological changes in vivo at pre-symptomatic stages. Recent evidences obtained from human samples and mouse models indicate the possibility to detect protein aggregates and other pathological features in the retina, paving the road for non-invasive rapid detection of $A D$ biomarkers. Here, we report the presence of amyloid beta plaques, tau tangles, neurodegeneration and detrimental astrocyte and microglia activation according to a disease associated microglia phenotype (DAM). Thus, we propose the human retina as a useful site for the detection of cellular and molecular changes associated with Alzheimer's disease.

Keywords: Alzheimer's disease, microglia, astrocytes, retina, beta-amyloid, tau, human, neurodegeneration

\section{INTRODUCTION}

Alzheimer's disease (AD) is a neurodegenerative disorder leading to dementia during elderly. This pathology is manifested with cognitive and psychiatric symptoms such as memory and cognitive impairments, behavioral abnormalities, disorientation and circadian rhythms disturbances (Hart et al., 2016). Due to population aging, $\mathrm{AD}$ cases are constantly increasing and it is estimated that by the year $2050 \mathrm{AD}$ will globally affects about 115 million people (Alzheimer's Disease International World Alzheimer Report, 2009; Scheltens et al., 2016) thus posing a growing concern on public health. 
Studies from post-mortem brains revealed that, at the neuropathological level, distinctive features of $\mathrm{AD}$ include aggregation of amyloid beta protein $(A \beta)$ and tau protein hyperphosphorylation (pTau), which cause synapses loss and neuronal degeneration. These plaques and tangles are generally associated with activated microglia and reactive astrocytes in $\mathrm{AD}$ brain (Ahmed et al., 2017; Henstridge et al., 2019). Over the years several candidate drugs have been proposed as a putative therapy for AD. However, despite many attempts, a definitive cure has not been found yet and only symptomatic treatments are available.

A possible explanation for this arises from the fact that $\mathrm{AD}$ is a complex multifactorial disorder, while pharmacological treatments are preferentially directed against a specific cellular target. Moreover, proteins aggregation can occur decades before the appearance of neurological symptoms, thus making interventions at later stages less effective.

It is therefore tempting to speculate that $\mathrm{AD}$ diagnosis at earlier stages, possibly when synapses and neuronal functions are not yet compromised, could give better results in terms of pharmacological and clinical intervention (Frisoni et al., 2017).

The search for early biomarkers in $\mathrm{AD}$ has become a very attractive area of study. Imaging brain changes in $A \beta$ with techniques such as computed tomography (CT), magnetic resonance index (MRI) and positron emission tomography (PET) in combination with the analysis of cerebrospinal fluid (CSF) have established $\mathrm{A} \beta$ and tau as an indicator of the AD disease.

In particular, both PET and CSF biomarkers allow to diagnose $\mathrm{AD}$ during the prodromal phase of the disease (Rowe et al., 2013; Petersen et al., 2016), but pitfalls such as high cost and invasiveness make these tests unsuitable for a populationwide screening.

Recently, researchers focused their interests on the retina in order to find new biomarkers for $\mathrm{AD}$. The rationale behind this choice rely on the fact that, the retina and the brain share a common embryological origin therefore they could share pathological mechanisms as well (Sernagor et al., 2001; Kavcic et al., 2011; Chang et al., 2014; Lee et al., 2014; Bambo et al., 2015; Javaid et al., 2016). Recent studies have shown that anatomical alterations such as thinning of the ganglion cell and retinal nerve fiber layers can be detected already during $\mathrm{AD}$ early stages (Thomson et al., 2005; Paquet et al., 2007) thus strengthening the idea that the retina could be used in early $\mathrm{AD}$ diagnosis. However, far from reaching a consensus, studies on retinal tissues from $\mathrm{AD}$ patients and mouse models produced contrasting results. For instance, amyloid plaques have been found in $\mathrm{AD}$ post-mortem retinas (but not in control cases) by Löffler et al. (1995). Similar results have been confirmed by other research groups (Hoh Kam et al., 2010; Alexandrov et al., 2011; Koronyo-Hamaoui et al., 2011; Koronyo et al., 2017), in addition a positive correlation between inclusion body and cortical amyloid burden has been observed (Snyder et al., 2016). On the contrary, den Haan and collegues detected a signal for phosphorilated Tau (pTau) in the inner and outer plexiform layer of the retina (IPL and OPL respectively), but they did not find any signal for $A \beta$ plaques and neurofibrilary tangles (den Haan et al., 2018). Most likely, these discrepancies may be attributed to differences in staining methods, sections preparation and time of tissue harvesting. Moreover, restricting retinal analysis to the presence of protein aggregates and vascular alterations solely may be poorly specific for $\mathrm{AD}$ diagnosis, as $\mathrm{A} \beta$ deposits have been found also in other pathological conditions such as macular degeneration (Zhao et al., 2015). Beyond classical neuropathological hallmarks, proteomic analysis of cerebrospinal fluid from $\mathrm{AD}$ patients revealed the upregulation of proteins related to microglia and astrocytes activation. For instance, $\mathrm{A} \beta$ plaques aggregation can activate astrocytes and microglia thus inducing the release of mediators of inflammation such as interleukin-1 beta (IL-1 $\beta$ ) and ultimately cellular and neuronal apoptosis both in brain and retina (Liu et al., 2014).

In neurodegenerative disease, microglia response consists in migrating to sites of damage or injury, secreting numerous inflammatory molecules, and phagocytizing debris and aggregated proteins (Heneka et al., 2015; Song and Colonna, 2018). However, microglia, astrocytes and immune signaling are not just secondary players in disease processes but actually contribute to synaptic and neuron loss and buildup of pathogenic proteins even at the earliest stages of disease (Hong et al., 2016; Shi et al., 2017; Sosna et al., 2018). Aggregation of A $\beta$ plaques can lead to inflammatory reactions, astrocytes and microglia activation, release of inflammatory cytokines such as interleukin1 beta (IL-1 $\beta$ ) and cellular and neuronal apoptosis in both the brain and retina (Liu et al., 2014). Moreover, the misregulated expression of glia released soluble factors in $\mathrm{AD}$ has been linked to disease associated microglia, and to microglia induced NLRP3 inflammasome and complement activation (Heneka et al., 2013; Sheedy et al., 2013; Lian et al., 2015; Iram et al., 2016) and synaptic loss (Hong et al., 2016). Moreover, a specific microglia phenotype has been described in neurodegenerative diseases characterized by the downregulation of microglia homeostatic genes, the upregulation of specific degeneration associated markers (DAM), and sustained by the upregulation of TREM2 (Yeh et al., 2017; Deczkowska et al., 2018).

To gain a better understanding and to identify new molecular targets for a more complete panel of putative biomarkers for $\mathrm{AD}$ diagnosis, we performed immunostaining on human retinas obtained from both $\mathrm{AD}$ patients and age matched controls. We report the presence of $\mathrm{A} \beta$ and pTau protein aggregates together with neuronal loss. We also report, for the first time, the upregulation of IL1- $\beta$ on microglia and the presence of neurotoxic $\mathrm{A} 1$ astrocytes in AD retina. These findings highlight protein aggregates and cellular markers as targets to be considered for $\mathrm{AD}$ diagnosis.

\section{MATERIALS AND METHODS}

\section{Human Samples}

Human retinal slices from AD patients and age matched controls were purchased from Human Eye Biobank for Research, St Michael Hospital, Toronto, Canada. Prior to death, donors signed informed consent for autopsy and use of tissue and medical records for research purposes. No documented history of eye disease was reported for the $\mathrm{AD}$ or control cases. The use of 
human tissue has been approved by the Ethics Committee of Fondazione Santa Lucia I.R.C.C.S. to GR.

\section{Immunofluorescence}

Slices were deparaffined with absolute Xylene $(3 \times 5 \mathrm{~min})$ and rehydrated by scaling down from $100 \%$ to $50 \%$ ethanol, $2 \times 10 \mathrm{~min}$ for each concentration. After PBS washes $(3 \times 5 \mathrm{~min})$, antigen retrieval has been performed by soaking slices for $40 \mathrm{~min}$ in a warm solution containing (in $\mathrm{mM}$ ): $10 \mathrm{Na}$-citrate, $0.05 \%$ Tween $20, \mathrm{pH} 6.0,90^{\circ} \mathrm{C}$. Subsequently, slices were incubated for $45 \mathrm{~min}$ in a blocking solution (3\% goat serum and $0.3 \%$ Triton X-100 in PBS). Primary antibodies diluted in blocking solution were incubated over-night at $4^{\circ}$ [anti- $\beta$ Tubulin III, T2200, Sigma, clone Aa441-450, 1:500; anti-GFAP, MAB360, Millipore, clone GA5; 1:400; anti-Iba1, 019-19741, Wako, 1:400; antiOPN, 691302, Biolegend, clone A15059B, 1:100; Anti- $\beta$ Amyloid, 8243s, CELL SIGNALING, clone D54D2, 1:100; anti-CleavedCasapase3, 9661s, CELL SIGNALING, clone Asp175, 1:100; antiPhosphoTau (Thr212, Ser214), MN106, INVITROGEN clone AT100 1:50; anti-PhosphoTau (Ser 202, Thr205), INVITROGEN, clone AT8, 1:40; anti-C3d, A0063, Dako, 1:500; anti-IL-1 3 , sc-32294, Santa Cruz Biotechnology, clone E7-2-hIL1 $\beta$, 1:50]. After three washes in PBS, sections were stained for $45 \mathrm{~min}$ with a secondary antibody and Hoechst in order to visualize nuclei, then coverslips were mounted with Diamond Antifade Mountant (Molecular Probes) and images acquired with a confocal microscopy.

\section{Confocal Spinning Disk and VCS Microscopy}

The acquisition of the images was performed through a Nikon Eclipse Ti equipped with a X-Light V2 spinning disk combined with a VCS (Video Confocal Super resolution) module (CrestOptics) based on structured illumination and with a LDI laser source ( 89 North). The images were acquired by using Metamorph software version 7.10.2. (Molecular Devices) with a 60x PlanApo $\lambda$ oil objective (1.4 numerical aperture) and sectioning the slice in $Z$ with a step size of $0.2 \mu \mathrm{m}$ for spinning disk and $0.15 \mu \mathrm{m}$ for VCS to obtain a total Z-stack of about $10 \mu \mathrm{m}$. In order to achieve super-resolution, raw data obtained by the VCS module have been processed with a modified version of the joint Richardson-Lucy (jRL) algorithm (Ingaramo et al., 2014; Ströhl and Kaminski, 2015; Chakrova et al., 2016), where the out of focus contribution of the signal has been explicitly added in the image formation model used in the jRL algorithm, and evaluated as a pixel-wise linear "scaled subtraction" (Heintzmann and Benedetti, 2006) of the raw signal. The acquisitions obtained were transformed into a z-projection and then analyzed using the ImageJ software.

\section{Laser Scanning Acquisitions}

For conventional confocal laser scanning analysis of retinal slices, images acquisition has been performed through a confocal laser scanning microscope (microscope (FV10i Olympus) equipped with a $60 \mathrm{x}$ water immersion objective. Acquired images were processed and analyzed off line using ImageJ. For each retina six images were acquired. For every image the maximum intensity projections of z-series stacks was created.

\section{Microglia Density Analysis}

The number of $\mathrm{Iba1}^{+}$microglia cells has been reported as number of somas per acquired area $\left(400 \mu \mathrm{m}^{2}\right)$. To quantify microglia density, the same images were analyzed by Metamorph software. A z-projection based on the maximal intensity signal was obtained and after threshold setting, fluorescence intensity value has been recorded. Data are expressed as area occupied by fluorescent cells versus total slice area.

\section{Astrogliosis Analysis}

Astrogliosis analysis has been performed by staining retinas section for GFAP and C3. Following threshold adjustment astrogliosis was quantified as fluorescence intensity above threshold. Data are expressed as area occupied by fluorescent cells versus total slice area. For the measurement of GFAP/C3 colocalization, the value of GFAP-overlapping the C3 signal was considered.

\section{Analysis of Neurodegeneration}

Confocal images of cells positive for cleaved caspase 3 , an effector enzyme of the apoptotic pathway, were analyzed by means of Metamorph software. We manually counted the number of cells positive for the signal of the cleaved caspase 3 within each image. Thereafter, this number has been divided by the total number of ganglion neurons counted in the region of interest (ROI) and thus expressed as percentage of degenerating ganglion cells in each slice.

\section{FISH}

Sections were deparaffined with absolute Xylene $(3 \times 5 \mathrm{~min})$ and partially rehydrated with $70 \%$ ethanol $(2 \times 10$ min each $)$ before proceeding with RNA FISH staining. RNA in situ hybridization was performed as described previously (Raj and Tyagi, 2010) with minor modifications: briefly, sections were incubated in Wash Buffer A (Stellaris, Biosearch Technologies) for $5 \mathrm{~min}$ and then, in the Hybridization Buffer supplemented with 2 mM VRC complex (Sigma, R3380) and $125 \mathrm{nM}$ TREM2 FISH probes $3^{\prime}$-end labeled with Quasar 670 fluorophore (Biosearch Technologies, SMF-1063-5). Incubation was performed overnight at $37^{\circ} \mathrm{C}$ in Top Brite automatic slide hybridizer (Resnova). After two washes in Wash buffer A for 30 min and one wash with Wash Buffer B for $5 \mathrm{~min}$ at $37^{\circ} \mathrm{C}$, Hoechst was added for $15 \mathrm{~min}$ at room temperature. Finally, slices were mounted with ProLong Diamond Antifade Mountant (Thermo Fischer Scientific Pm36961) and analyzed with a confocal laser scanning microscope. Immunofluorescence for IBA1 was performed sequentially to RNA FISH staining.

\section{Protein Aggregates}

The size of the amyloid plaques and tau tangles was studied on images acquired with the confocal microscope and subsequently analyzed with the Metamorph program (version 7.6.5.0). The "Trace Region" function of this program makes it possible to 
surround the deposits of these proteins and to obtain their volume expressed in $\mu \mathrm{m}^{3}$. Their density has been studied with the Image J program through which it was possible to count the number of these aggregates within each slice analyzed.

\section{Statistics and Data Analysis}

Data are shown as the Mean \pm SEM. Statistical significance between controls and AD patients was assessed with the nonparametric Mann-Whitney test or T-test as indicated. A $p$ value $<0.05$ was considered significant. All statistical analyses were done using Sigma Plot 11.0, Origin or Clampfit software.

\section{RESULTS}

\section{Typical $A \beta$ and pTau Protein Aggregates in AD Human Retina}

The accumulation of $A \beta$ and pTau aggregates in the retinal tissue has been reported on both AD human tissue (Koronyo-Hamaoui et al., 2011; Koronyo et al., 2017) and mouse models (Criscuolo et al., 2018; Grimaldi et al., 2018), thus suggesting a putative use of the eye as a valuable structure for the study and the diagnosis of $\mathrm{AD}$ and other neurodegenerative disease. However, due to the complex nature of the disease, and to the positive $A \beta$ staining in macular degeneration (Lynn et al., 2017), a more comprehensive panel of biomarkers needs to be defined to avoid misleading $\mathrm{AD}$ diagnosis. Using human retinal slices from $\mathrm{AD}$ patients and controls (samples were obtained from the St. Michael's Hospital Human Eye Biobank), we first confirmed the presence of $A \beta$ and pTau aggregates in the retinal layers.

We analyzed retinal cross sections from 10 clinically and neuropathologically confirmed AD patients (mean age \pm SEM: $85.7 \pm 2.7$ years; range $71-98$ years; 8 females and 2 males) and 10 healthy controls (mean age \pm SEM: $75.3 \pm 2.9$ years; range: $65-$ 93 years; 6 females and 4 male). In all AD patients we detected retinal $A \beta$ immunoreactivity and deposits both in the inner and outer layers (minimum diameter: $110 \mathrm{~nm}$; Figures 1A,B). $\mathrm{A} \beta$ staining, evaluated as $\mathrm{A} \beta$ plaques number in a region of $400 \mu \mathrm{m}^{2}$, was significantly higher in $\mathrm{AD}$ patients $(10.4 \pm 1.8$ $n=45 / 5$ fields/patients) compared to controls $(3.8 \pm 0.7 n=44 / 5$ fields/controls; $p<0.01$; Figure 1C). Sparse and diffuse retinal A $\beta$ deposits were found occasionally also in control slices (Figure 1A, left), as previously reported (Koronyo et al., 2017). Plaques volume measured on 3D stacks was higher in $\mathrm{AD}$ patients respect to controls as reported in the histogram in Figure 1D.

In order to assess the presence of hyperphosphorylated tau isoforms we performed immunofluorescence analysis using two different monoclonal antibodies directed against the hyperphosphorylated tau protein (pTau; clone AT- 8 and clone AT-100, Figure 1E). Following the staining with the AT-100 clone we observed a strong and diffuse staining for pTau both in the IPL and OPL in the majority of AD patients (6/10). Indeed, pTau immunoreactivity was significantly higher in the AD retina compared to age matched controls as quantified by fluorescence intensity in each field of view (AD: $0.76 \pm 0.11, n=38 / 6$ fields/patients; CTRL: $0.22 \pm 0.06, n=32 / 6$ fields/controls; $p<0.005 ; \mathrm{FOV}=400 \mu \mathrm{m}^{2}$; Figure 1F), thus confirming the accumulation of neurofibrillary tangles deposits in $\mathrm{AD}$ patients retina (Figure 1E, right). Using the AT-8 clone we found discrete pTau immunofluorescence in the IPL and in the OPL only in two out of 10 patients analyzed; in these AD patients the number of pTau tangles was significantly higher respect to control (AD: $4.4 \pm 1.7, n=15 / 2$ fields/patients; CTRL: $0.9 \pm 0.4 ; n=15 / 2$ fields/controls; $p<0.05$; not shown). Co-labeling the retina with AT-100 and TUJ1 as neuronal marker indicated that in AD retina pTau expression was mainly in the Retinal Ganglion Cells (RGC) of the inner layer (IL; Figure 1G).

These findings clearly demonstrate an increase in $\mathrm{A} \beta$ and $\mathrm{pTau}$ aggregates in the retina of $\mathrm{AD}$ patients respect to controls thus pointing at them as putative biomarkers for $\mathrm{AD}$ diagnosis.

\section{AD Human Retina Displays Neurodegeneration in the Ganglion Cell Layer}

An increased cleavage of proteins such as APP and presenilins operated by caspase-3, has been associated with neurodegeneration in AD (Louneva et al., 2008). In a previous paper from our laboratory, we reported immunoreactivity for the cleaved caspase- 3 at the level of the RGC layer of the 3xTg-AD mouse model (Grimaldi et al., 2018). A positive staining for caspase-3 is also present in the IL of human retinas (Figure $2 \mathbf{A}$, green dots) in TUJ1 positive cells (red). In particular we found that the number of caspase-3 positive retinal ganglion cells in each field of view (FOV) examined was increased compared to age matched controls (AD: $11.4 \pm 2.2 \%, n=17 / 6$ fields/patients; CTRL: $6.0 \pm 1.0 \%, n=18 / 6$ fields/control; $p<0.05$; Figure 2B) These results indicated that the $\mathrm{AD}$ retinal IL is more subject to neuronal death thus suggesting that visual defects and optic nerve thinning observed in $\mathrm{AD}$ may rely on retinal ganglion cells neurodegeneration.

\section{Increased Astrocytosis and Microglia Reactivity in AD Patients Retina}

In $\mathrm{AD}$ brain, astrocytes have been found closely associated with fibrillar amyloid plaques suggesting that $\mathrm{A} \beta$ accumulation may serves as a cue for the activation of this cell type (Henstridge et al., 2019). Astrocytes activation can have a neuroprotective effect but it may trigger the release of pro-inflammatory species such as cytokines and chemokines (Stadelmann et al., 2002; Farina et al., 2007). To evaluate putative astrogliosis in the retina of AD patients we performed immunostaining for the GFAP. Analysis of acquired images showed a marked astrogliosis localized at the level of the ganglion cell layer. Astrogliosis may arise also as a consequence of aging however, the amount of astrocyte activation was more pronounced in the $\mathrm{AD}$ retina compared to controls (Figure 3A), as quantified by fluorescence intensity in each field of view (AD: $5.5 \pm 1.1, n=44 / 6$ fields/patients; CTRL: $1.9 \pm 0.3$, $n=44 / 6$ fields/controls; $p<0.001$; Figure 3B).

As for astrocytes, also microglia activation can show a dual role in inflammatory processes. Increased microglia reactivity in the retina has been observed in post-mortem tissues from $\mathrm{AD}$ patients (Beach et al., 1989) and in the 3xTg-AD mouse model (Grimaldi et al., 2018). Immunostaining against Iba1 a 
A

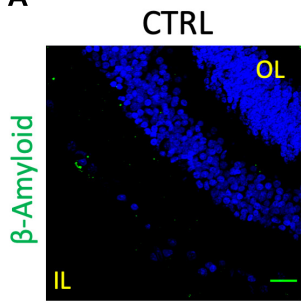

E

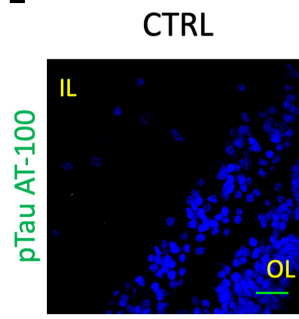

B

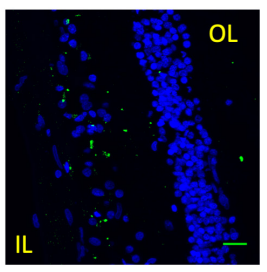

$A D$

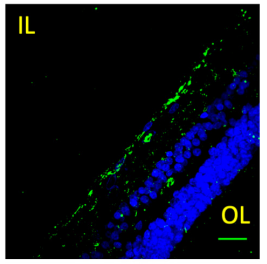

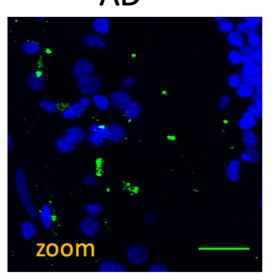

$\mathbf{F}$

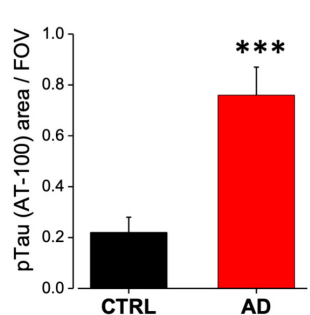

C

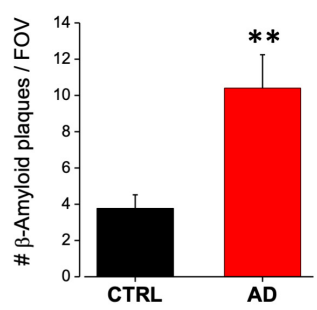

D

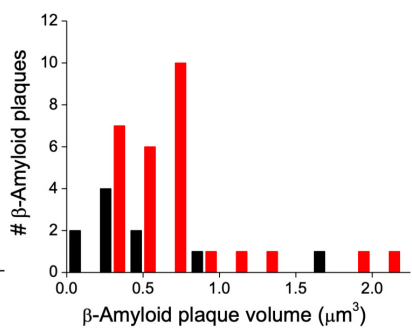

G

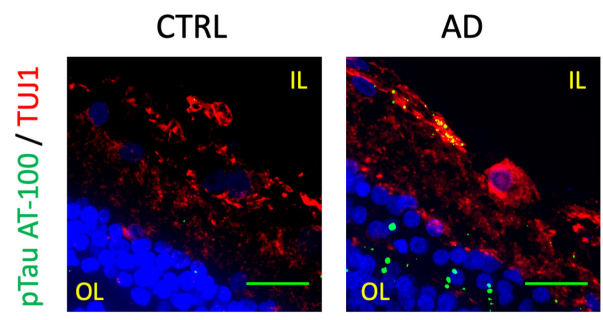

FIGURE 1 | Human AD retina displays $\beta$-amyloid and pTau aggregates. (A) Representative images of retinal slices from AD patients and control cases immunolabeled with anti- $\beta$-amyloid antibody (green) and Hoechst for nuclei visualization (blue); bar $20 \mu \mathrm{m}$. (B) Insert representing $\beta$-amyloid staining in AD retina at higher magnification; bar $20 \mu \mathrm{m}$ (C) Number of $\beta$-amyloid plaques/field of view (** $p<0.01$ AD vs. Ctrl; $t$-test; $n=45 / 5$ fields/patients). (D) Distribution of $\beta$-amyloid plaque volume measured in AD patients and control cases. (E) Representative images of retinal slices from AD patients and control cases immunolabeled with anti-p-tau AT-100 antibody (green) and Hoechst for nuclei visualization (blue); bar $20 \mu \mathrm{m}$. (F) Quantification of p-tau AT-100 area covered by fluorescent signal/field of view (*** $p<0.001 \mathrm{AD}$ vs. Ctrl; $t$-test; $n=38 / 6$ fields/patients). (G) Representative images of retinal slices from AD patients and control immunolabeled with anti-p-tau AT-100 (green) and anti-TUJ1 as RGC marker (red) at higher magnification (Hoechst for nuclei visualization in blue; bar 20 $\mu$ m). IL, inner layer; OL, outer layer.
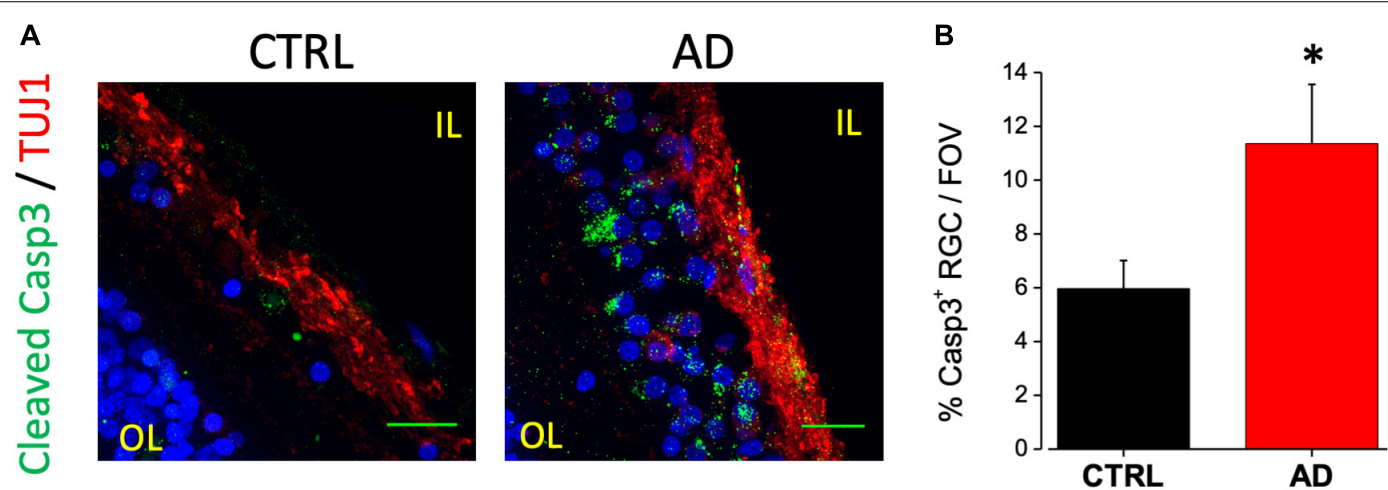

FIGURE 2 | Ganglion cell neuron degeneration in AD patient's retina. (A) Representative images of retinal slices from AD patients and control cases immunolabeled with anti-cleaved caspase-3 antibody (green), anti-TUJ1 as RGC marker (red) and Hoechst for nuclei visualization (blue); bar 20 $\mu$ m. (B) Bar chart representing the percentage of RGC neuron positive for cleaved caspase-3 on each field of view ( ${ }^{*} p<0.05$ AD vs. Ctrl; $t$-test; $n=17 / 4$ fields/patients). IL, inner layer; OL, outer layer.

microglia marker, revealed that this cell type was mainly present in two layers: the inner plexiform and the outer plexiform layers (Figure 3C). Microglia cell density was increased in $\mathrm{AD}$ patients retina compared to age matched controls (AD: $3.9 \pm 0.3 ; n=73 / 10$ fields/patients; CTRL: $2.5 \pm 0.3, n=73 / 10$ fields/controls; $p<0.005$; Figure 3D).

These results, confirming the presence of altered astrocyte and microglia density in human $\mathrm{AD}$ retina, suggest the possibility to add markers for glial activation in the set of retinal biomarkers for $\mathrm{AD}$ diagnosis.

\section{AD Human Retina Displays Upregulation of Neurotoxic Microglia and Astrocytes}

Several different proteins have been demonstrated to be involved in the interplay among neurons, astrocytes and microglia in neurodegenerative diseases. Particularly, altered expression of these proteins in $\mathrm{AD}$ has been linked to disease associated microglia, to microglia induced detrimental astrocytes activation (Heneka et al., 2013; Sheedy et al., 2013) and synaptic loss (Hong et al., 2016). Here we examined and compared the expression of IL-1 $\beta$, TREM-2 (triggering receptor expressed on myeloid 

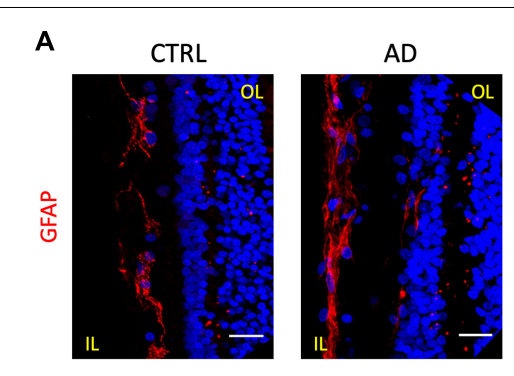

B
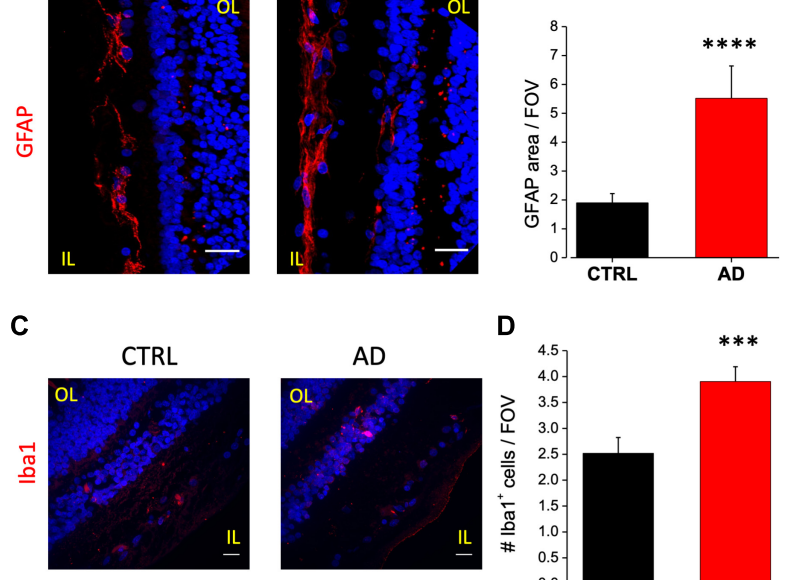

D

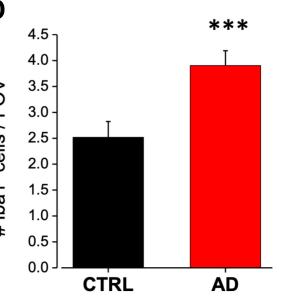

FIGURE 3 | Increased astrocytes and microglia cell density in the retina of $A D$ patients. (A) Representative images of retinal slices from AD patients and control cases immunolabeled with anti-GFAP antibody (red) and Hoechst for nuclei visualization (blue); bar $20 \mu \mathrm{m}$. (B) Quantification of GFAP area covered by fluorescent signal/field of view $\left({ }^{* * * *} p<0.001\right.$ AD vs. Ctrl; $t$-test; $n=44 / 6$ fields/patients). (C) Representative images of retinal slices from AD patients and control cases immunolabeled with anti-lba1 antibody (red) and Hoechst for nuclei visualization (blue); bar $20 \mu \mathrm{m}$. (D) Quantification of Iba1 area covered by fluorescent signal/field of view $\left({ }^{* * *} p<0.005\right.$ AD vs. Ctrl; $t$-test; $n=73 / 10$ fields/patients). IL, inner layer; OL, outer layer.

cells-2), complement component C3 and OPN (Osteopontin) between $\mathrm{AD}$ and control samples.

Immunofluorescence analysis of IL- $1 \beta$ expression on $\mathrm{AD}$ retinal slices showed enhanced IL- $1 \beta$ cytoplasmic staining in the inner nuclear layer (INL) of $\mathrm{AD}$ patients compared to age matched controls (Figure 4A, left). IL-1 $\beta$ immunostaining in AD patients retina was found to be colocalized with Iba1-expressing cells, a marker of microglia, but not with the astrocytes marker GFAP (not shown). Indeed, co-labeling with Ibal (Figure 4A, right) revealed that the number of microglia cells positive for IL$1 \beta$ was significantly higher compared to control (AD: $3.5 \pm 0.4$, $n=31 / 5$ fields/patients; CTRL: $2.2 \pm 0.4, n=29 / 5$ fields/controls; $p<0.05$; Figure 4B; average number of Iba1/IL- $1 \beta+$ cells for each FOV), with almost all microglia cells expressing IL$1 \beta$ in $\mathrm{AD}$.

Astrocytic C3 upregulation has been demonstrated to be downstream of the microglia induced IL1- $\beta$ activation. Moreover, its expression seems to be regulated by $\beta$-amyloid in both $\mathrm{AD}$ mouse models and human brain (Lian et al., 2015). C3 upregulation is now considered to be a marker for detrimental A1 astrocytic phenotype taking part in $\mathrm{AD}$ related synaptic loss. We examined C3 expression in human retina by immunofluorescence analysis. We found C3 to be expressed in the ganglion cell layer. Co-staining with GFAP revealed that C3 immunoreactivity was confined in GFAP positive cells as shown in Figure 4C. Fluorescence intensity quantification showed that the percentage of $\mathrm{C} 3$ positive astrocytes was strongly upregulated in $\mathrm{AD}$ retina respect to control ( $\mathrm{AD}: 26 \pm 4$, $n=45 / 6$ fields/patients; CTRL: $7.8 \pm 1.6, n=46 / 6$ fields/controls; $p<0.001$; Figure 4D).

Upregulation of OPN in AD patients CSF may arise from neurons, microglia or both. Indeed, while the OPN encoding gene SPP1 (secreted phosphoprotein 1) is now considered a good DAM microglia marker (Ulland et al., 2017), AD brains display OPN upregulation in CA1 pyramidal neurons (Wung et al., 2007). We analyzed the expression level of OPN in human retinal slices of $\mathrm{AD}$ patients and age matched controls by immunofluorescence analysis using a monoclonal antibody against the full length OPN peptide (Figure 5A, left). We found significant upregulation of OPN expression in AD retinas, quantified as fluorescence intensity over threshold in each field of view $\left(400 \mu \mathrm{m}^{2}\right)(\mathrm{AD}: 3.0 \pm 0.6, n=24 / 3$ fields/patients) compared to age matched controls (CTRL: $0.7 \pm 0.2, n=27 / 4$ fields/patients; $p<0.001$ ) (Figure 5B). OPN expression was localized in the retinal ganglion cell layer as demonstrated by the co-staining against tubulin isoform $\beta$ III (TUJ1; Figure $\mathbf{5 A}$, middle), a selective marker for RGC neurons. Indeed, the percentage of RGC neurons positive for OPN (Figure 5A, left) was significantly higher in $\mathrm{AD}$ respect to control retinas (AD: $84 \pm 5 \%, n=36 / 6$ fields/patients; CTRL: $63 \pm 6 \%, n=34 / 6$ fields/patients; $p<0.01$; Figure 5C). Conversely, co-labeling with anti-Iba1 did not show upregulated OPN expression on microglia (data not shown).

TREM2 is now considered a crucial regulator in promoting microglia responses to $\mathrm{A} \beta$ in $\mathrm{AD}$. TREM 2 transcript has been shown to be over-expressed by microglia both in the brain of AD mouse models as well as human patients (Frank et al., 2008; Lue et al., 2015; Wang et al., 2015), suggesting that TREM2 upregulation could mirror with $\mathrm{AD}$ progression. Moreover, in the 3xTg-AD mouse model, we reported the upregulation of TREM2 mRNA in sorted retinal microglia cells (Grimaldi et al., 2018). Due to the lack of specific antibodies against human TREM2, FISH experiments have been performed in order to evaluate the expression level of TREM2 transcript in retinal slices of $\mathrm{AD}$ patients and age matched controls (Figure 5D). The analysis of TREM2 RNA levels obtained with fluorescence threshold analysis did not show, on average, any difference between AD and control retinas (AD: $0.18 \pm 0.03, n=31 / 4$ fields/patients; CTRL: $0.22 \pm 0.06, n=37 / 4$ fields/controls; $p=0.3$; data not shown). However, the number of TREM2 positive cells in each field of view $\left(400 \mu \mathrm{m}^{2}\right)$ was higher in $\mathrm{AD}$ retina respect to control (AD: $8.5 \pm 0.8, n=31 / 4$ fields/patients; CTRL: $6.2 \pm 0.6, n=37 / 4$ fields/controls; $p<0.05$; Figure 5E), indicating an increased TREM-2 expression. When TREM2 FISH analysis was performed together with immunofluorescence for Iba1, we observed Ibal positive cells expressing TREM2 in AD retina (Figure 5D, left). It should be noticed, however, that in our experiments TREM2 staining was not exclusively confined to Iba1-positive cells. This could be ascribed to protein denaturation taking place during FISH protocol, preventing an absolute quantification of TREM2 expressing retinal microglia cells.

These data indicate that detrimental astrocytic and microglia activation can be detected in $\mathrm{AD}$ patients retina with increased release of pro-inflammatory compounds that could be considered as possible biomarkers for $\mathrm{AD}$ diagnosis. 

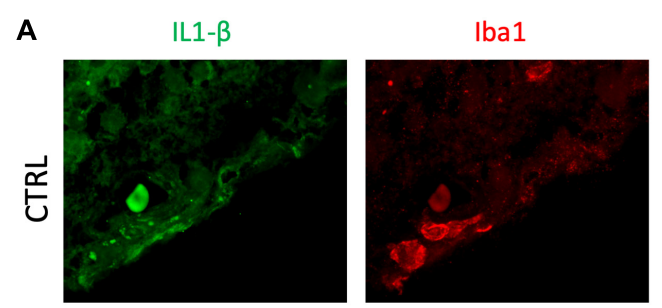

IL1- $\beta /$ Iba1
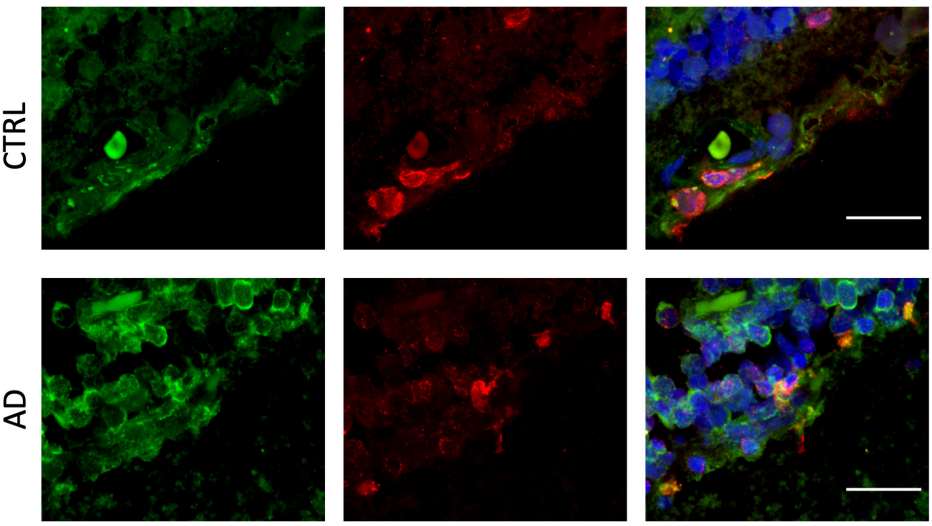

C

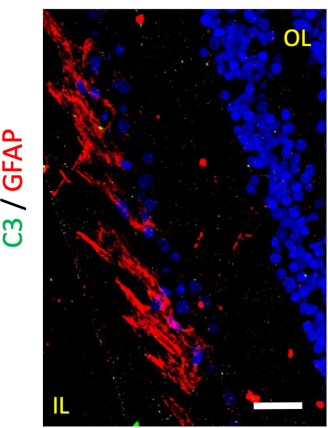

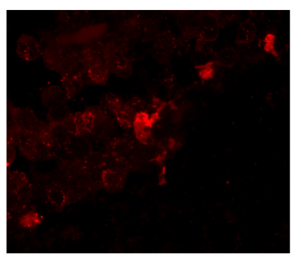

$A D$

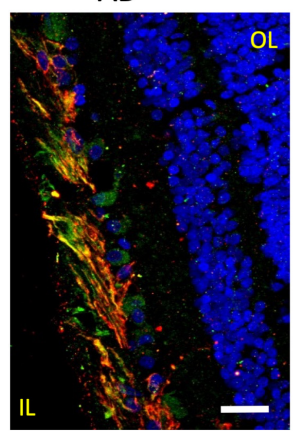

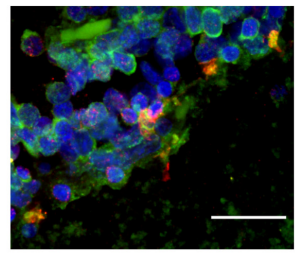

D
B

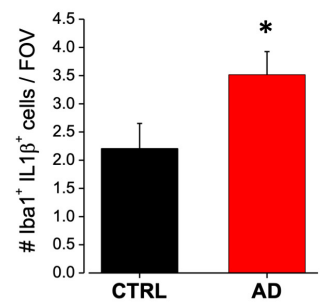

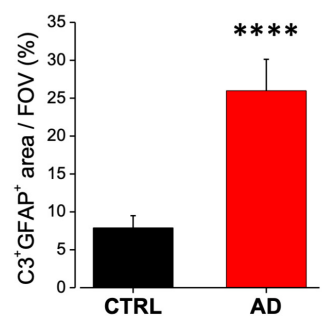

FIGURE 4 | AD patient's retina display complement C3 and IL-1 $\beta$ upregulation. (A) Representative images of retinal slices from AD patients (bottom) and control cases (top) immunolabeled with anti-IL-1 $\beta$ antibody (green), Iba1 (red) and Hoechst for nuclei visualization (blue); bar $20 \mu \mathrm{m}$. (B) Quantification of cells positive for both Iba1 and IL-1 $\beta$ signal/field of view ( ${ }^{*} p<0.05$ AD vs. Ctrl; $t$-test; $n=31 / 5$ fields/patients). (C) Representative images of retinal slices from AD patients and control cases immunolabeled with anti-GFAP antibody (red), C3 (green) and Hoechst for nuclei visualization (blue); bar $20 \mu \mathrm{m}$. (D) Quantification of GFAP/C3 co-localization area/field of view ${ }^{* * * *} p<0.001$ AD vs. Ctrl; $t$-test; $n=45 / 6$ fields/patients). IL, inner layer; OL, outer layer.

\section{DISCUSSION}

As of now, a definitive $\mathrm{AD}$ diagnosis is made possible only after post-mortem examination of brain tissue (Elahi and Miller, 2017). Despite many attempts there is an urgent need to find new easy-to-acquire, cost-effective strategies for $\mathrm{AD}$ diagnosis, even at early stages in order to allow a timely and effective therapeutic program (Frisoni et al., 2017).

The retinal tissue is now considered as a very promising structure to be used in searching of new AD biomarkers as it is thought that it could mirror the pathological changes happening in the brain during the disease progression. Even more, the retina is an accessible structure and this anatomical feature could be of extreme importance in the development of new imaging methods for its examination.

However, $A \beta$ and pTau protein aggregates could not be considered specific markers to $\mathrm{AD}$ therefore, a more comprehensive panel of biomarkers is needed.

For this reason, we used post-mortem retinal slices from $\mathrm{AD}$ patients to investigate the presence of classical $\mathrm{AD}$ features and the expression of specific neuron-to-glia signaling proteins found both in the CSF of $\mathrm{AD}$ patients and in the brain of $\mathrm{AD}$ mouse models (Doens and Fernández, 2014). We here report that $\mathrm{AD}$ patients retina show, in addition to the presence of $\mathrm{A} \beta$ plaques and pTau tangles, ganglion neuron degeneration, astrogliosis, microglia activation, and up-regulation of specific disease associated neuron-to-glia signaling proteins, such as IL$1 \beta, \mathrm{C} 3, \mathrm{OPN}$ and TREM2.

In agreement with previous results (Koronyo et al., 2017; den Haan et al., 2018), we observed increased A $\beta$ deposits and diffuse spreading of pTAu signals in the inner retinal layer of AD patients compared to age-matched controls. However, it has to be noticed that only a subset of $\mathrm{AD}$ retinal slices here analyzed were positive for pTau staining with AT- 8 and AT-100 antibodies, this could be ascribed to pTau specific topographic distribution in human retina; indeed pTau staining was clearly reported in the anterior part of the superior retina (Koronyo et al., 2017; den Haan et al., 2018), and using transverse retinal slices obtained from the Human Eye Bank we could not asses which part of the retina we were analyzing. Due to this technical issue it is plausible that we are underestimating the amount of pTau in the $\mathrm{AD}$ patients retina. Also, we show that neuronal apoptosis is significantly higher in the retina of $\mathrm{AD}$ patients compared to age matched controls, predominantly in RGC layer.

As it is well known that glial cells are activated in $\mathrm{AD}$ associated inflammatory states, we performed immunostaining to 

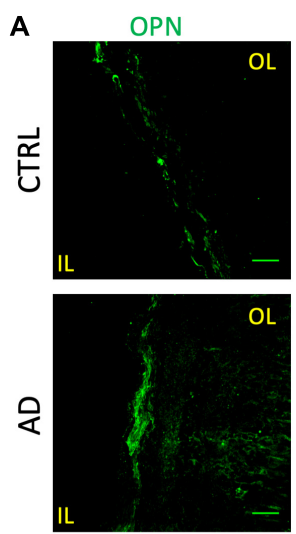

D
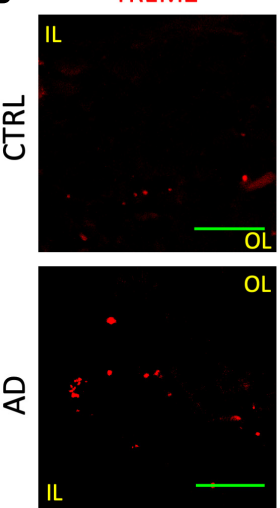
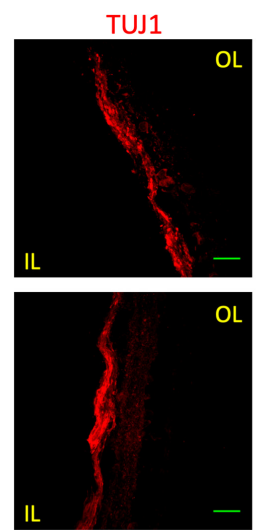

Iba1
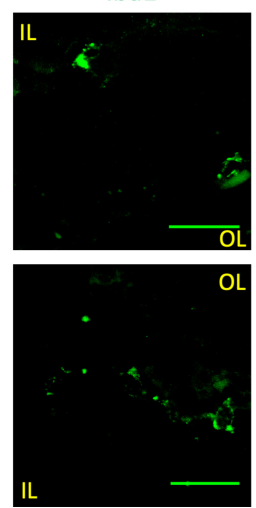

OPN / TUJ1
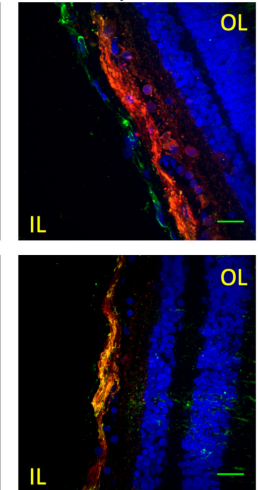

B

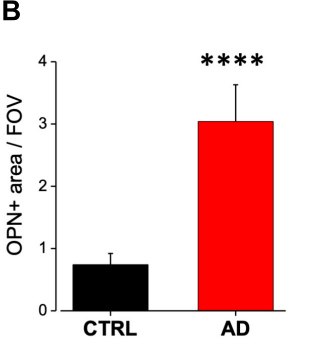

C

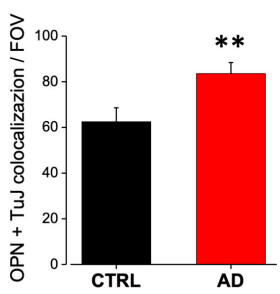

Iba1 / TREM2
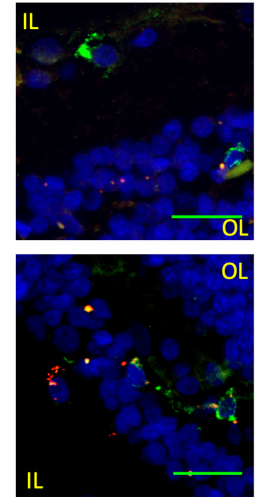

E

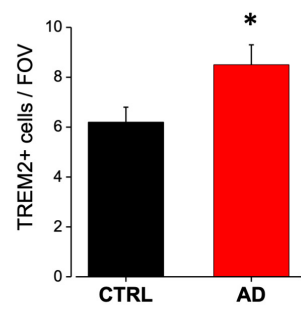

FIGURE 5 | Osteopontin and TREM2 expression in AD patient's retina. (A) Representative images of retinal slices from AD patients (bottom) and control cases (top) immunolabeled with anti-OPN (green), tuj-1 (red) and Hoechst for nuclei visualization (blue); bar $20 \mu \mathrm{m}$. (B) Quantification of OPN area covered by fluorescent signal/field of view (**** $p<0.001$ AD vs. Ctrl; $t$-test; $n=24 / 3$ fields/patients). (C) Quantification of OPN/TUJ1 co-localization area/field of view, in\% (** $p<0.01$ AD vs. Ctrl; $t$-test; $n=36 / 6$ fields/patients). (D) Representative images of retinal slices from AD patients (bottom) and control cases (top) stained with anti TREM2 mRNA fluorescent probe (red), anti-lba1 (green) and Hoechst for nuclei visualization (blue); bar $20 \mu \mathrm{m}$. Note that cells expressing TREM2 are not always positive for Iba1. (E) Number of TREM2 positive cells/field of view ( ${ }^{*} p<0.05$ AD vs. Ctrl; $t$-test; $n=31 / 4$ fields/patients). IL, inner layer; OL, outer layer.

evaluate putative difference between controls and AD samples. Although GFAP and Ibal reactivity was present both in $\mathrm{AD}$ and aged controls, the area of GFAP positive staining and the number of microglia cells were significantly higher in AD retinas thus indicating that glia (both astrocytes and microglia) activation is significantly more pronounced in $\mathrm{AD}$ retinas compared to controls.

We show here for the first time that retinal microglia of $\mathrm{AD}$ patients display, respect to their age matched controls, higher expression of IL-1 $\beta$ a typical marker of pro-inflammatory and DAM microglia (Keren-Shaul et al., 2017) suggesting microglia response to $A \beta$ and pTau accumulation. These results are in line with the increased expression of IL- $1 \beta$ found in brain microglia in human patients and mouse models (as reviewed in Shaftel et al., 2008). Conversely, while in the brain of 5xFAD mouse model IL- $1 \beta$ was overexpressed by astrocytes (Rosenzweig et al., 2019), in human AD and control retina we did not observe IL$1 \beta$ expression in GFAP positive cells. It should be noted that we found enhanced IL-1 $\beta$ staining also in other cells in the INL of $A D$ retina, probably due to the NLRP3 inflammasome activation and monocytes infiltration.
We also report an increase of TREM2 mRNA level in the retina of $\mathrm{AD}$ patients respect to aged controls. The importance of TREM2 in the central nervous system (CNS) is widely recognized, as TREM2 mutations are linked to an increased risk of developing several neurodegenerative diseases (Ulland and Colonna, 2018), and TREM2 RNA has been found to be upregulated in the brain of patients and mouse models (Keren-Shaul et al., 2017). Moreover, we previously reported TREM2 upregulation in retinal microglia sorted from early symptomatic 3xTg-AD mice (Grimaldi et al., 2018). However, while it is evident that TREM2 expression on microglia cells plays a prominent role in driving microgliosis in the brain of AD mouse models and patients (Keren-Shaul et al., 2017), we did not find increased expression of TREM2 transcript on Ibal positive cells in the retina of $\mathrm{AD}$ patients. It can be speculated that in our samples the expression of TREM2 on microglia cells is underestimated due to technical reasons. Indeed, RNA FISH experiments on post-mortem paraffinembedded tissue is per se technically challenging, requiring the use of formamide to increase the hybridization efficiency (Cattoretti et al., 1993; Gilbert et al., 2007). This procedure might 
induce protein denaturation causing the failure of subsequent immunofluorescence analysis. Another possible explanation may rely on the age of $\mathrm{AD}$ patients here analyzed. Indeed, while microglia TREM2 expression is important in triggering and sustaining the DAM phenotype in response to protein aggregates during disease progression (Song and Colonna, 2018; Ulland and Colonna, 2018), downregulation of TREM2 has been reported in human post-mortem retinal tissues of patients suffering from Age-Related Macular Degeneration (Bhattacharjee et al., 2016) where $A \beta$ deposits are present. In this framework it is likely that post mortem retinal tissue from aged patients is not the ideal specimen for TREM2 analysis. Moreover, the observed increase in TREM2 transcript level could be due to infiltrating monocytes negative for Ibal, as reported in the brain of $\mathrm{AD}$ patients (Fahrenhold et al., 2018).

The analysis of osteopontin expression in retinal tissue revealed increased $\mathrm{OPN}$ staining in $\mathrm{AD}$ retinal ganglion cells with evident colocalization with tubulin $\beta$-III. This result is partially in contrast with previous findings in the $\mathrm{AD}$ brain. Indeed, although OPN transcript (SPP1) expression has been shown to be upregulated in microglia in AD models (Ulland et al., 2017) and thus considered a marker for DAM phenotype, we did not find OPN upregulation on Ibal positive cells. On the other hand our result is in line with data reporting that the protein osteopontin is upregulated on CA1 hippocampal pyramidal neurons in human AD brain (Wung et al., 2007). Moreover, in mouse retina, OPN is reported to be expressed on RGC (Ju et al., 2000; Duan et al., 2015). These considerations make OPN a good candidate biomarker for retinal AD diagnosis despite its cellular localization.

Here, for the first time we report the presence of A1 astrocytic phenotype in the retina of $\mathrm{AD}$ patients, as revealed by the strong upregulation of $\mathrm{C} 3$ protein on retinal astrocytes. This result is consistent with the reported presence of $\mathrm{A} 1$ reactive brain astrocytes in neurodegenerative diseases (Liddelow et al., 2017). The importance of reactive A1 astrocytes in neurodegenerative diseases rely on the evidence that synaptic loss can be favored by element of the complement cascade released by the A1 astrocytes (Stevens et al., 2007; Hong et al., 2016; Sekar et al., 2016). It is noteworthy that $\mathrm{A} 1$ activation may be initiated by microglia released IL-1 $\beta$ as well as by extracellular $A \beta$ accumulation (Lian et al., 2015; Liddelow and Barres, 2017), both found and here reported in retina of $\mathrm{AD}$ patients.

These observations further support the possibility that ocular biomarkers could be used for early detection of AD associated neurodegeneration. It should be noted, however, that other ocular pathologies, such as glaucoma shares histopathological hallmarks with $\mathrm{AD}$ including increased levels of tau protein and microglial activation (Ramirez et al., 2017). It is therefore desirable that a

\section{REFERENCES}

Ahmed, M. E., Iyer, S., Thangavel, R., Kempuraj, D., Selvakumar, G. P., Raikwar, S. P., et al. (2017). Co-localization of glia maturation factor with nlrp3 inflammasomeand autophagosome markers in human Alzheimer's disease brain. J. Alzheimers. Dis. 60, 1143-1160. doi: 10.3233/JAD- 170634 complete panel of biomarkers able to discriminate between agerelated and disease-related retinal changes would be available and could be used as a target for in vivo imaging through a retinal scan. However, this will requires the development of both specific $\mathrm{AD}$ biomarkers ligands and long working distance high resolution imaging techniques, in order to achieve a non-invasive and inexpensive diagnosis of $\mathrm{AD}$ through the retinal scan.

\section{DATA AVAILABILITY}

The datasets generated for this study are available on request to the corresponding author.

\section{ETHICS STATEMENT}

Human Subject Research: The studies involving human participants were reviewed and approved by the Ethics Committees Fondazione Santa Lucia IRCCS. Written informed consent for participation was not required for this study in accordance with the national legislation and the institutional requirements.

\section{AUTHOR CONTRIBUTIONS}

AG, NP, and FO designed, carried out, and analyzed the immunofluorescence experiments on protein aggregates and neurodegeneration. MR and RP designed, carried out, and analyzed the immunofluorescence experiments on astrocytes and microglia. MG designed, carried out, and analyzed the VCS and spinning disks acquisition. TS and AG designed, performed, and analyzed the FISH experiments. SD wrote the manuscript with the help of RP, GR, DR, and CL. SD conceived the project.

\section{FUNDING}

This work was supported by the CrestOptics-IIT JointLab for Advanced Microscopy to AG, RP, GR, and SD, the MARBEL Life2020 grant to SD, and the SynaNet H2020 Program to AG, $\mathrm{CL}, \mathrm{SD}$, and DR.

\section{ACKNOWLEDGMENTS}

The authors wish to thank the Imaging Facility at Center for Life Nano Science (IIT@Sapienza) for the support and technical advice.

Alexandrov, P. N., Pogue, A., Bhattacharjee, S., and Lukiw, W. J. (2011). Retinal amyloid peptides and complement factor $\mathrm{H}$ in transgenic models of Alzheimer's disease. Neuroreport 22, 623-627. doi: 10.1097/WNR.0b013e3283497334

Alzheimer's Disease International World Alzheimer Report, (2009). Alzheimer's Disease International World Alzheimer Report. London: Alzheimer's Disease Internationa. 
Bambo, M. P., Garcia-Martin, E., Otin, S., Pinilla, J., Larrosa, J. M., Polo, V., et al. (2015). "Visual function and retinal nerve fibre layer degeneration in patients with Alzheimer disease: correlations with severity of dementia. Acta Ophthalmol. 93, e507-e508. doi: 10.1111/aos.12635

Beach, T. G., Walker, R., and McGeer, E. G. (1989). Patterns of gliosis in Alzheimer's disease and aging cerebrum. Glia 2, 420-436. doi: 10.1002/glia.440020605

Bhattacharjee, S., Zhao, Y., Dua, P., Rogaev, E. I., and Lukiw, W. J. (2016). microRNA-34a-Mediated Down-regulation of the microglial-enriched triggering receptor and phagocytosis-sensor TREM2 in age-related macular degeneration. PLoS One 11:e0150211. doi: 10.1371/journal.pone.0150211

Cattoretti, G., Piler, S., Parravicini, C., Becker, M. H. G., Poggi, S., Bifulco, C., et al. (1993). Antigen unmasking on formalin-fixed, paraffin-embedded tissue sections. J. Pathol. 171, 83-98. doi: 10.1002/path.1711710205

Chakrova, N., Rieger, B., and Stallinga, S. (2016). Deconvolution methods for structured illumination microscopy. J. Opt. Soc. Am. A Opt. Image Sci. Vis. 33, B12-B20. doi: 10.1364/JOSAA.33.000B12

Chang, L. Y. L., Lowe, J., Ardiles, A., Lim, J., Grey, A. C., Robertson, K., et al. (2014). Alzheimer's disease in the human eye. Clinical tests that identify ocular and visual information processing deficit as biomarkers. Alzheimer's Dement. 10, 251-261. doi: 10.1016/j.jalz.2013.06.004

Criscuolo, C., Cerri, E., Fabiani, C., Capsoni, S., Cattaneo, A., and Domenici, L. (2018). The retina as a window to early dysfunctions of Alzheimer's disease following studies with a 5xFAD mouse model. Neurobiol. Aging. 67, 181-188. doi: 10.1016/j.neurobiolaging.2018.03.017

Deczkowska, A., Keren-Shaul, H., Weiner, A., Colonna, M., Schwartz, M., and Amit, I. (2018). Disease-associated microglia: a universal immune sensor of neurodegeneration. Cell. 173, 1073-1081. doi: 10.1016/j.cell.2018.05.003

den Haan, J., Morrema, T. H. J., Verbraak, F. D., de Boer, J. F., Scheltens, P., Rozemuller, A. J., et al. (2018). Amyloid-beta and phosphorylated tau in postmortem Alzheimer's disease retinas. Acta Neuropathol. Commun. 2018 6:147. doi: 10.1186/s40478-018-0650-x

Doens, D. D., and Fernández, P. L. (2014). Microglia receptors and their implications in the response to amyloid $\beta$ for Alzheimer's disease pathogenesis. J. Neuroinflamm. 11:48. doi: 10.1186/1742-2094-11-48

Duan, X., Qiao, M., Bei, F., Kim, I. J., He, Z., and Sanes, J. R. (2015). Subtypespecific regeneration of retinal ganglion cells following axotomy: effects of osteopontin and mTOR signaling. Neuron 85, 1244-1256. doi: 10.1016/j. neuron.2015.02.017

Elahi, F. M., and Miller, B. L. (2017). A clinicopathological approach to the diagnosis of dementia. Nat. Rev. Neurol. 13, 457-476. doi: 10.1038/nrneurol. 2017.96

Fahrenhold, M., Rakic, S., Classey, J., Brayne, C., Ince, P. G., Nicoll, J. A. R., et al. (2018). MRC-CFAS. TREM2 expression in the human brain: a marker of monocyte recruitment? Brain Pathol. 28, 595-602. doi: 10.1111/bpa. 12564

Farina, C., Aloisi, F., and Meinl, E. (2007). Astrocytes are active players in cerebral innate immunity. Trends Immunol. 28, 138-145. doi: 10.1016/j.it.2007.01.005

Frank, S., Burbach, G. J., Bonin, M., Walter, M., Streit, W., Bechmann, I., et al. (2008). TREM2 is upregulated in amyloid plaque-associated microglia in aged APP23 transgenic mice. Glia 56, 1438-1447. doi: 10.1002/glia. 20710

Frisoni, G. B., Boccardi, M. F., Barkhof, F., Blennow, K., Cappa, S., Chiotis, K., et al. (2017). Strategic roadmap for an early diagnosis of Alzheimer's disease based on biomarkers. Lancet Neurol. 16, 661-676. doi: 10.1016/S1474-4422(17)30159-X

Gilbert, M. T. P., Haselkorn, T., Bunce, M., Sanchez, J. J., Lucas, S. B., Jewell, L. D., et al. (2007). The isolation of nucleic acids from fixed, paraffin-embedded tissues-which methods are useful when? PLoS One. 2:e537. doi: 10.1371/ journal.pone.0000537

Grimaldi, A., Brighi, C., Peruzzi, G., Ragozzino, D., Bonanni, V., Limatola, C., et al. (2018). Inflammation, neurodegeneration and protein aggregation in the retina as ocular biomarkers for Alzheimer's disease in the 3xTg-AD mouse model. Cell Death Dis. 9:685. doi: 10.1038/s41419-018-0740-5

Hart, N. J., Koronyo, Y., Black, K. L., and Koronyo-Hamaoui, M. (2016). Ocular indicators of Alzheimer's: exploring disease in the retina. Acta Neuropathol. 132, 767-787. doi: 10.1007/s00401-016-1613-6

Heintzmann, R., and Benedetti, P. A. (2006). High-resolution image reconstruction in fluorescence microscopy with patterned excitation. Appl. Opt. 10, 50375045 .
Heneka, M. T., Carson, M. J., El Khoury, J., Landreth, G. E., Brosseron, F., and Feinstein, D. L. (2015). Neuroinflammation in Alzheimer's disease. Lancet Neurol. 14, 388-405. doi: 10.1016/S1474-4422(15)70016-5

Heneka, M. T., Kummer, M. P., Stutz, A., Delekate, A., Schwartz, S., Vieira-Saecker, A., et al. (2013). NLRP3 is activated in Alzheimer's disease and contributes to pathology in APP/PS1 mice. Nature 493, 674-678. doi: 10.1038/nature11729

Henstridge, C. M., Hyman, B. T., and Spires-Jones, T. L. (2019). Beyond the neuron-cellular interactions early in Alzheimer disease pathogenesis. Nat. Rev. Neurosci. 20, 94-108. doi: 10.1038/s41583-018-0113-1

Hoh Kam, J., Lenassi, E., and Jeffery, G. (2010). Viewing ageing eyes: diverse sites of amyloid Beta accumulation in the ageing mouse retina and the up-regulation of macrophages. PLoS One 5:e13127. doi: 10.1371/journal.pone.0013127

Hong, S., Beja-Glasser, V. F., Nfonoyim, B. M., Frouin, A., Li, S., Ramakrishnan, S., et al. (2016). Complement and microglia mediate early synapse loss in Alzheimer mouse models. Science 352, 712-716. doi: 10.1126/science.aad8373

Ingaramo, M., York, A. G., Hoogendoorn, E., Postma, M., Shroff, H., and Patterson, G. H. (2014). Richardson-Lucy deconvolution as a general tool for combining images with complementary strengths. Chemphyschem 15, 794-800. doi: 10. 1002/cphc.201300831

Iram, T., Trudler, D., Kain, D., Kanner, S., Galron, R., Vassar, R., et al. (2016). Astrocytes from old Alzheimer's disease mice are impaired in $A \beta$ uptake and in neuroprotection. Neurobiol. Dis. 96, 84-94. doi: 10.1016/j.nbd.2016. 08.001

Javaid, F. Z., Brenton, J., Guo, L., and Cordeiro, M. F. (2016). Visual and ocular manifestations of Alzheimer's disease and their use as biomarkers for diagnosis and progression. Front. Neurol. 19:55. doi: 10.3389/fneur.2016.00055

Ju, W. K., Kim, K. Y., Cha, J. H., Kim, I. B., Lee, M. Y., Oh, S. J., et al. (2000). Ganglion cells of the rat retina show osteopontin-like immunoreactivity. Brain Res. 852, 217-220. doi: 10.1016/s0006-8993(99)02140-x

Kavcic, V., Vaughn, W., and Duffy, C. J. (2011). Distinct visual motion processing impairments in aging and Alzheimer's disease. Vision Res. 51, 386-395. doi: 10.1016/j.visres.2010.12.004

Keren-Shaul, H., Spinrad, A., Weiner, A., Matcovitch-Natan, O., Dvir-Szternfeld, R., Ulland, T. K., et al. (2017). A Unique Microglia Type Associated with Restricting Development of Alzheimer's Disease. Cell 169, 1276.e17-1290.e17. doi: 10.1016/j.cell.2017.05.018

Koronyo, Y., Biggs, D., Barron, E., Boyer, D. S., Pearlman, J. A., Au, W. J., et al. (2017). Retinal amyloid pathology and proof-of-concept imaging trial in Alzheimer's disease. JCI Insight 2:93621. doi: 10.1172/jci.insight.93621

Koronyo-Hamaoui, M., Koronyo, Y., Ljubimov, A. V., Miller, C. A., Ko, M. K., Black, K. L., et al. (2011). Identification of amyloid plaques in retinas from Alzheimer's patients and noninvasive in vivo optical imaging of retinal plaques in a mouse model. Neuroimage 54(Suppl. 1), S204-S217. doi: 10.1016/j. neuroimage.2010.06.020

Lee, D.-H., Laemmer, A. B., Waschbisch, A., Struffert, T., Maihöfner, C., Schwab, S., et al. (2014). Neuromyelitis optica presenting with relapses under treatment with natalizumab: a case report. J. Med. Case Rep. 8.

Lian, H., Yang, L., Cole, A., Sun, L., Chiang, A. C., Fowler, S. W., et al. (2015). $\mathrm{NF \kappa B}$-activated astroglial release of complement C3 compromises neuronal morphology and function associated with Alzheimer's disease. Neuron. 85, 101-115. doi: 10.1016/j.neuron.2014.11.018

Liddelow, S. A., and Barres, B. A. (2017). Reactive Astrocytes: Production. Function, and Therapeutic Potential. Immunity 2017 46, 957-967. doi: 10.1016/ j.immuni.2017.06.006

Liddelow, S. A., Guttenplan, K. A., Clarke, L. E., Bennett, F. C., Bohlen, C. J., Schirmer, L., et al. (2017). Neurotoxic reactive astrocytes are induced by activated microglia. Nature. 541, 481-487. doi: 10.1038/nature21029

Liu, R. T., Wang, A., To, E., Gao, J., Cao, S., Cui, J. Z., et al. (2014). Vinpocetine inhibits amyloid-beta induced activation of NF-кB, NLRP3 inflammasome and cytokine production in retinal pigment epithelial cells. Exp. Eye Res. 127, 49-58. doi: 10.1016/j.exer.2014.07.003

Löffler, K. U., Edward, D. P., and Tso, M. O. (1995). Immunoreactivity against tau, amyloid precursor protein, and beta-amyloid in the human retina. Invest. Ophthalmol. Vis. Sci. 36, 24-31.

Louneva, N., Cohen, J. W., Han, L. Y., Talbot, K., Wilson, R. S., Bennett, D. A., et al. (2008). Caspase-3 is enriched in postsynaptic densities and increased in Alzheimer's disease Am. J. Pathol. 173, 1488-1495. doi: 10.2353/ajpath.2008. 080434 
Lue, L. F., Schmitz, C. T., Serrano, G., Sue, L. I., Beach, T. G., and Walker, D. G. (2015). TREM2 protein expression changes correlate with Alzheimer's disease neurodegenerative pathologies in post-mortem temporal cortices. Brain Pathol. 25, 469-480. doi: 10.1111/bpa.12190

Lynn, S. A., Keeling, E., Munday, R., Gabha, G., Griffiths, H., Lotery, A. J., et al. (2017). The complexities underlying age-related macular degeneration: could amyloid beta play an important role? Neural Regen. Res. 12, 538-548. doi: 10.4103/1673-5374.205083

Paquet, C., Boissonnot, M., Roger, F., Dighiero, P., Gil, R., and Hugon, J. (2007). Abnormal retinal thickness in patients with mild cognitive impairment and Alzheimer's disease. Neurosci. Lett. 420, 97-99. doi: 10.1016/j.neulet.2007. 02.090

Petersen, R. C., Wiste, H. J., Weigand, S. D., Rocca, W. A., Roberts, R. O., Mielke, M. M. Jr., et al. (2016). Association of elevated amyloid levels with cognition and biomarkers in cognitively normal people from the community AMA. Neurol 73, 85-92. doi: 10.1001/jamaneurol.2015.3098

Raj, A., and Tyagi, S. (2010). Detection of individual endogenous RNA transcripts in situ using multiple singly labeled probes. Methods Enzymol. 472, 365-386. doi: 10.1016/S0076-6879(10)72004-8

Ramirez, A. I., de Hoz, R., Salobrar-Garcia, E., Salazar, J. J., Rojas, B., Ajoy, D., et al. (2017). The Role of microglia in retinal neurodegeneration: Alzheimer's Disease, parkinson, and glaucoma. Front. Aging Neurosci. 9:214. doi: 10.3389/ fnagi.2017.00214

Rosenzweig, N., Dvir-Szternfeld, R., Tsitsou-Kampeli, A., Keren-Shaul, H., BenYehuda, H., Weill-Raynal, P., et al. (2019). PD-1/PD-L1 checkpoint blockade harnesses monocyte-derived macrophages to combat cognitive impairment in a tauopathy mouse model. Nat. Commun. 10:465. doi: 10.1038/s41467-01908352-5

Rowe, C. C., Bourgeat, P., Ellis, K. A., Brown, B., Lim, Y. Y., Mulligan, R., et al. (2013). Predicting Alzheimer disease with $\beta$-amyloid imaging: results from the Australian imaging, biomarkers, and lifestyle study of ageing. Ann. Neurol. 74, 905-913. doi: 10.1002/ana.24040

Scheltens, P., Blennow, K., Breteler, M. M., de Strooper, B., Frisoni, G. B., and Salloway, S. (2016). Alzheimer's disease. Lancet 388, 505-517. doi: 10.1016/ S0140-6736(15)01124-1

Sekar, A., Bialas, A. R., de Rivera, H., Davis, A., Hammond, T. R., Kamitaki, N., et al. (2016). Schizophrenia risk from complex variation of complement component 4. Nature 530, 177-183. doi: 10.1038/nature16549

Sernagor, E., Eglen, S. J., and Wong, R. O. (2001). Development of retinal ganglion cell structure and function. Prog. Retin. Eye Res. 20, 139-174. doi: 10.1016/ s1350-9462(00)00024-0

Shaftel, S. S., Griffin, W. S., and O'Banion, M. K. (2008). The role of interleukin1 in neuroinflammation and Alzheimer disease: an evolving perspective. J. Neuroinflamm. 5:7. doi: 10.1186/1742-2094-5-7

Sheedy, F. J., Grebe, A., Rayner, K. J., Kalantari, P., Ramkhelawon, B., Carpenter, S. B., et al. (2013). CD36 coordinates NLRP3 inflammasome activation by facilitating intracellular nucleation of soluble ligands into particulate ligands in sterile inflammation. Nat. Immunol. 14, 812-820. doi: 10.1038/ni. 2639

Shi, Y., Yamada, K., Liddelow, S. A., Smith, S. T., Zhao, L., Luo, W., et al. (2017). ApoE4 markedly exacerbates tau-mediated neurodegeneration in a mouse model of tauopathy. Nature 549, 523-527. doi: 10.1038/nature24016

Snyder, P. J., Johnson, L. N., Lim, Y. Y., Santos, C. Y., Alber, J., Maruff, P., et al. (2016). Nonvascular retinal imaging markers of preclinical Alzheime"s disease. Alzheimers Dement. 1, 169-178. doi: 10.1016/j.dadm.2016.09.001

Song, W. M., and Colonna, M. (2018). The identity and function of microglia in neurodegeneration. Nat. Immunol. 19, 1048-1058. doi: 10.1038/s41590-0180212-1
Sosna, J., Philipp, S., and Albay, R. III (2018). Early long-term administration of the CSF1R inhibitor PLX3397 ablates microglia and reduces accumulation of intraneuronal amyloid, neuritic plaque deposition and pre-fibrillar oligomers in 5XFAD mouse model of Alzheimer's disease. Mol. Neurodegener. 13:11. doi: 10.1186/s13024-018-0244-x

Stadelmann, C., Kerschensteiner, M., Misgeld, T., Brück, W., Hohlfeld, R., Lassmann, H., et al. (2002). BDNF and gp145trkB in multiple sclerosis brain lesions: neuroprotective interactions between immune and neuronal cells? Brain. 125(Pt 1), 75-85. doi: 10.1093/brain/awf015

Stevens, B., Allen, N. J., Vazquez, L. E., Howell, G. R., Christopherson, K. S., Nouri, N., et al. (2007). The classical complement cascade mediates CNS synapse elimination. Cell. 14, 1164-1178. doi: 10.1016/j.cell.2007.10.036

Ströhl, F., and Kaminski, C. F. (2015). A joint richardson-lucy deconvolution algorithm for the reconstruction of multifocal structured illumination microscopy data. Methods Appl. Fluoresc. 3:014002. doi: 10.1088/2050-6120/3/ $1 / 014002$

Thomson, K. L., Yeo, J. M., Waddell, B., Cameron, J. R., and Pal, S. (2005). A systematic review and meta-analysis of retinal nerve fiber layer change in dementia, using optical coherence tomography. Alzheimers Dement. 1, 136143. doi: 10.1016/j.dadm.2015.03.001

Ulland, T. K., and Colonna, M. (2018). TREM2 - a key player in microglial biology and Alzheimer disease. Nat. Rev. Neurol. 14, 667-675. doi: 10.1038/s41582-0180072-1

Ulland, T. K., Song, W. M., Huang, S. C., Ulrich, J. D., Sergushichev, A., Beatty, W. L., et al. (2017). TREM2 maintains microglial metabolic fitness in Alzheimer's Disease. Cell. 17, 649.e13-663.e13. doi: 10.1016/j.cell.2017. 07.023

Wang, Y., Cella, M., Mallinson, K., Ulrich, J. D., Young, K. L., Robinette, M. L., et al. (2015). TREM2 lipid sensing sustains the microglial response in an Alzheimer's disease model. Cell. 160, 1061-1071. doi: 10.1016/j.cell.2015. 01.049

Wung, J. K., Perry, G., Kowalski, A., Harris, P. L., Bishop, G. M., Trivedi, M. A., et al. (2007). Increased expression of the remodeling- and tumorigenicassociated factor osteopontin in pyramidal neu- ons of the Alzheimer's disease brain. Curr. Alzheimer Res. 4, 67-72. doi: 10.2174/156720507779939869

Yeh, F. L., Hansen, D. V., and Sheng, M. (2017). TREM2, Microglia, and Neurodegenerative Diseases. Trends Mol. Med. 23, 512-533. doi: 10.1016/j. molmed.2017.03.008

Zhao, Y., Bhattacharjee, S., Jones, B. M., Hill, J. M., Clement, C., Sambamurti, K., et al. (2015). Beta-Amyloid precursor protein ( $\beta$ APP) processing in Alzheimer's Disease(AD) and age-related macular degeneration (AMD). Mol. Neurobiol. 52, 533-544. doi: 10.1007/s12035-014-8886-3

Conflict of Interest Statement: MG is currently employed at CrestOptics. This study received funding from the JointLab between Istituto Italiano di Tecnologia and CrestOptics.

The remaining authors declare that the research was conducted in the absence of any commercial or financial relationships that could be construed as a potential conflict of interest.

Copyright (c) 2019 Grimaldi, Pediconi, Oieni, Pizzarelli, Rosito, Giubettini, Santini, Limatola, Ruocco, Ragozzino and Di Angelantonio. This is an open-access article distributed under the terms of the Creative Commons Attribution License (CC BY). The use, distribution or reproduction in other forums is permitted, provided the original author(s) and the copyright owner(s) are credited and that the original publication in this journal is cited, in accordance with accepted academic practice. No use, distribution or reproduction is permitted which does not comply with these terms. 